\title{
Evaluación de desempeño de la mezcla asfáltica modificada con residuos de PVC tipo blíster
}

\section{Performance evaluation of modified asphalt mix with blister PVC waste}

\section{Nicole Cajina Cruz}

Asistente de investigación

Laboratorio Nacional de Materiales y Modelos Estructurales

Universidad de Costa Rica, Costa Rica

nico.cajina@gmail.com

\section{Edgar Camacho Garita}

Jefe de Laboratorio de Ensayos a Escala Natural

Laboratorio Nacional de Materiales y Modelos Estructurales

Universidad de Costa Rica, Costa Rica

edgar.camachogarita@ucr.ac.cr

\section{Alejandra Baldi}

Investigadora

Unidad de Investigación en Infraestructura y Transporte Laboratorio Nacional de Materiales y Modelos Estructurales Universidad de Costa Rica, Costa Rica

alejandra.baldi@ucr.ac.cr

\author{
José Pablo Aguiar Moya \\ Profesor asociado \\ Escuela de Ingeniería Civil \\ Universidad de Costa Rica, Costa Rica \\ jose.aguiar@ucr.ac.cr
}

Fecha de recepción: 17 de noviembre de 2020 / Fecha de aprobación: 08 de junio de 2021

\section{RESUMEN}

La modificación de mezclas asfálticas con polímeros de desecho brinda diversos beneficios. Entre los más importantes se encuentra la optimización del desempeño de la mezcla y el incremento en su vida útil. Esto sin considerar una reducción en la cantidad de residuos plásticos que no poseen un adecuado manejo de desecho.

El presente artículo muestra los resultados obtenidos de una investigación en donde se incorporó a una mezcla asfáltica un material de desecho tipo blíster (compuesto por polímeros como el PVC) por medio de la vía seca. Método en donde, previo a la adhesión del asfalto, se incorpora a la mezcla el modificante en sustitución de parte del agregado fino. Esto con el propósito de evaluar el desempeño de esta mezcla y comparar su comportamiento mecánico con el de una mezcla convencional.

Para la ejecución del proyecto se caracterizó el modificante (blíster) y se seleccionó el porcentaje adecuado para que el diseño de la mezcla modificada cumpliera con las especificaciones de la metodología Superpave. Con el diseño definido se realizaron los especímenes de ensayo y se evaluó su desempeño con las pruebas de: fatiga a cuatro puntos, rueda de Hamburgo, resistencia retenida a la tensión diametral y módulo dinámico. Como se identificó un adecuado comportamiento de la mezcla modificada, se evaluó su sensibilidad en una estructura de pavimento; identificando en qué capas de la mezcla asfáltica sería conveniente utilizarla.

Palabras clave: Mezcla asfáltica modificada, blíster, método seco, fatiga, deformación permanente, daño por humedad.

\section{ABSTRACT}

The modification of asphalt mix with waste polymers provides several benefits. Among the most important is the optimization of the performance of the mix and the increase in its useful life. Not to mention a reduction in the amount of plastic waste that does not have an adequate waste management.

This article shows the results obtained from a study where a blister type waste material (composed of polymers such as PVC) was incorporated into an asphalt mixture by the dry method. Method in which, prior to the asphalt adhesion, the modifier is incorporated to the mix in substitution of part of the fine aggregate. The purpose of the study was to evaluate the performance of the mix and compare its mechanical behavior with a conventional mix.

For the project development, the modifier was characterized, and the appropriate percentage was selected so that the design of the modified mixture would comply with Superpave requirements. With the optimized design the test specimens were prepared, and their performance was evaluated based on the following tests: four-point beam bending, Hamburg wheel, retained tensile strength (moisture damage) and dynamic modulus. Since an adequate behavior of the modified mix was observed, its sensitivity was evaluated in a pavement structure; identifying in which layers of the asphalt mix should the material be used.

Keywords: Modified asphaltic mix, blister, waste materials, fatigue, permanent deformation, moisture damage. 


\section{INTRODUCCIÓN}

Las mezclas asfálticas pueden presentar problemas en su desempeño debido a las propiedades de los materiales utilizados en el diseño, a las cargas aplicadas y a las condiciones climáticas a las que se exponen. Por lo que, al realizar el diseño de una mezcla, se deben considerar estos factores y procurar que la mezcla sea capaz de soportar la deformación permanente, el agrietamiento a fatiga y el daño por humedad.

La deformación permanente se asocia a las elevadas cargas de tránsito y a las altas temperaturas que experimenta el pavimento durante su vida útil (Mendoza et al., 2019). En cuanto al agrietamiento por fatiga, es provocado por las repeticiones de carga vehicular y suele presentarse en las áreas donde se perciben las mayores deflexiones con altas frecuencias, no se da una adecuada transferencia de las cargas hacia las capas inferiores del pavimento o no existe una óptima adherencia entre las capas (Muñoz, 2020).

Por otro lado, la disminución de adherencia entre el agregado y el asfalto producido por la presencia del agua en la mezcla se conoce como daño por humedad. Este deterioro puede manifestarse como una pérdida del recubrimiento del ligante asfáltico de la superficie del agregado (conocido como "stripping") o como un desprendimiento gradual de los agregados de la superficie de la mezcla (conocido como "raveling") (Caro y Álvarez, 2011).

Al no considerar los elementos anteriormente expuestos, se podrían presenciar deformaciones excesivas en la estructura. Cuando las mezclas asfálticas convencionales experimentan algún problema en su desempeño, es posible mitigarlo con metodologías alternativas de diseño, añadiendo aditivos o materiales de desecho. No obstante, al utilizar aditivos se pueden incrementar los costos de producción de la mezcla, por lo que emplear materiales de desecho se vuelve una opción más atractiva (Aguiar, Villegas, Loría y Salazar, 2013).

La incorporación de materiales de desecho se puede realizar por medio de la vía húmeda o la vía seca. El primer método, consiste en mezclar el material de desecho con el ligante asfáltico previo a su incorporación con los agregados y el segundo, en integrar el material con los agregados antes de añadir el asfalto (Rodríguez, 2016).
Rondón etal. (2009) mencionan que la modificación de mezclas asfálticas por medio de la adhesión de polímeros (producto de desechos industriales), es una técnica muy utilizada para mejorar propiedades mecánicas y reológicas de las mezclas convencionales, como lo son la susceptibilidad térmica, la rigidez y la resistencia al envejecimiento, a la deformación plástica y a la fatiga. Además de reducir el impacto ambiental que estos materiales generan (Rondón et al., 2009).

Por otro lado, el Programa de Naciones Unidas para el Desarrollo (PNUD) indica que en Costa Rica se desechan aproximadamente 550 t de plástico diario y únicamente el $9 \%$ es reciclado (Grajales, 2018). Además, aproximadamente el $10 \%$ de estos desechos son incinerados lo que puede generar emisiones de gases tóxicos, como ocurre con el PVC que libera cloruro de hidrógeno (Carranza et al., 2010).

Por lo que utilizar materiales derivados de los polímeros para la modificación de mezcla asfáltica, es una propuesta que promueve reutilizar este material y reducir la contaminación. Además, permite el cumplimiento de la Ley de la República de Costa Rica N9828 (Asamblea Legislativa, 2020), la cual indica que al menos el $50 \%$ del volumen total de los materiales utilizados en los proyectos de infraestructura vial del Estado, deben incluir algún porcentaje de materiales reutilizables.

En la investigación de Cajina (2020), se diseñó una mezcla asfáltica modificada con material de desecho tipo blíster por medio de la vía seca (sustitución de agregados). El blíster es un recipiente empleado en la industria farmacéutica, para almacenar medicamentos sólidos. Está compuesto por una lámina de polímero moldeada con cavidades, que son recubiertas con una lámina de aluminio.

Para la fabricación de los blísters se utilizan polímeros como polietileno (PE), el cloruro de polivinilideno (PVDC) y el cloruro de polivinilo (PVC), siendo este último el más empleado (Ávila, 2020). Ciertos de estos polímeros presentan dificultad para ser reciclados como el PVC que, por ejemplo, presenta contenidos elevados de cloro que dificultan el proceso de reciclaje (Camacho, 2013).

El objetivo principal del presente artículo es presentar un resumen de los hallazgos de la investigación de Cajina (2020), donde se evaluó el desempeño de una mezcla asfáltica modificada con material de desecho tipo blíster, a partir de una comparación del comportamiento mecánico de esta mezcla con el de una convencional. 


\section{METODOLOGÍA}

El diseño de la mezcla asfáltica modificada se basó en el de una mezcla convencional (control o patrón). Esta última fue elaborada por el LanammeUCR previo a la investigación, y tanto el diseño, como los resultados de desempeño, fueron brindados por esta institución para la ejecución del proyecto, de manera que fueran utilizados como parámetro de comparación (Lanamme, 2017).

Los diseños se realizaron según la metodología de diseño Superpave, basado en las propiedades volumétricas de la mezcla asfáltica: los vacíos de la mezcla (Va), los vacíos en el agregado mineral (VMA) y los vacíos rellenos de asfalto (VFA).

El asfalto y el agregado utilizado en las mezclas asfálticas fue caracterizado previamente por el Lanamme UCR. El asfalto fue el AC-30, con un grado de desempeño (PG) de 64-22. Y la fuente de agregado fue la proveniente del quebrador Río Claro (Zona Sur de Costa Rica), con un tamaño máximo nominal de $12,5 \mathrm{~mm}$.

En la Figura 1, se puede observar la curva granulométrica de los apilamientos definidos para el agregado de Río Claro siendo estos: agregado fino (2137-16), agregado intermedio A (2138-16), agregado intermedio B (2139-16) y agregado grueso (2140-16). Los números entre paréntesis indican la codificación asignada en el laboratorio a estas muestras.

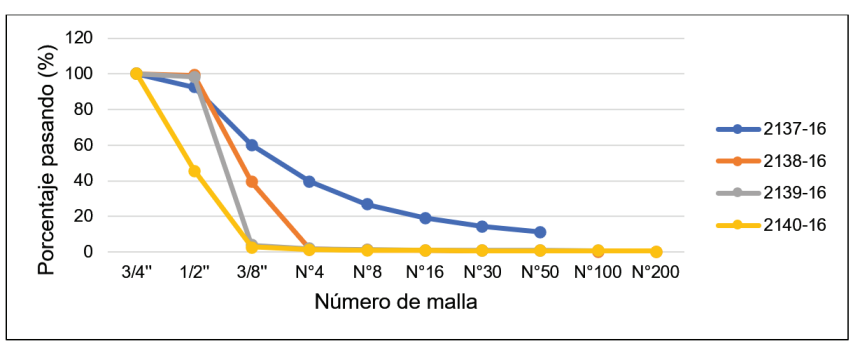

Figura 1. Granulometría de los agregados utilizados (Lanamme, 2016)

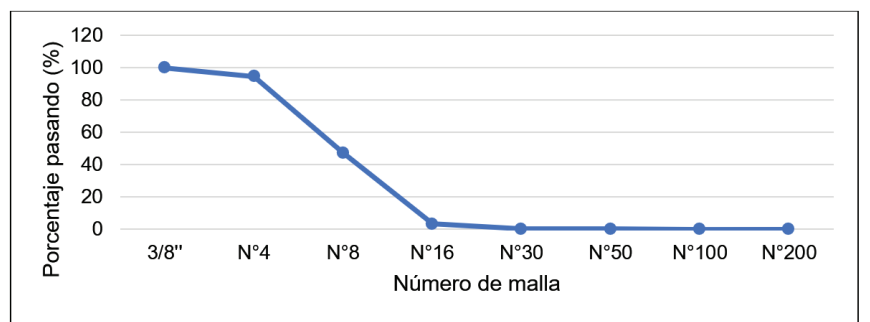

Figura 3. Granulometría del material de desecho tipo blíster

Para el caso de la mezcla asfáltica modificada, se incorporó el material de desecho tipo blíster (observar Figura 2), con una densidad de 1,401 g/mL, por medio de la vía seca. El 91,12\% de las partículas utilizadas en la mezcla, presentaron un tamaño entre la malla $\mathrm{N}^{\circ} 4(4,75 \mathrm{~mm})$ y la malla $\mathrm{N}^{\circ} 16(1,18 \mathrm{~mm})$ (ver Figura 3), permitiendo una mezcla uniforme y una trabajabilidad adecuada.

Como se mencionó anteriormente, la modificación se dio por medio de la vía seca, en la cual se sustituye parte del agregado fino por el material modificante. Por lo que, una vez caracterizado el material, se procedió a elegir el porcentaje óptimo del blíster, el cual se determinó según el porcentaje de diseño teórico de vacíos de la metodología Superpave $(4 \pm 1) \%$. Para esto, se realizaron tres mezclas con distintos porcentajes de blíster (en peso de agregado seco): 0,5\%, 1,0 \% y $1,5 \%$. Y cada una con un porcentaje de asfalto por peso total de la mezcla (\% PTM) de 6,32 \%; mismo porcentaje utilizado en el diseño de la mezcla control, lo que permitió una mejor comparación entre las mezclas.

En el Cuadro 1 se muestran las propiedades volumétricas de la mezcla control y de las tres mezclas modificadas, además del cumplimiento de las especificaciones de la metodología Superpave.

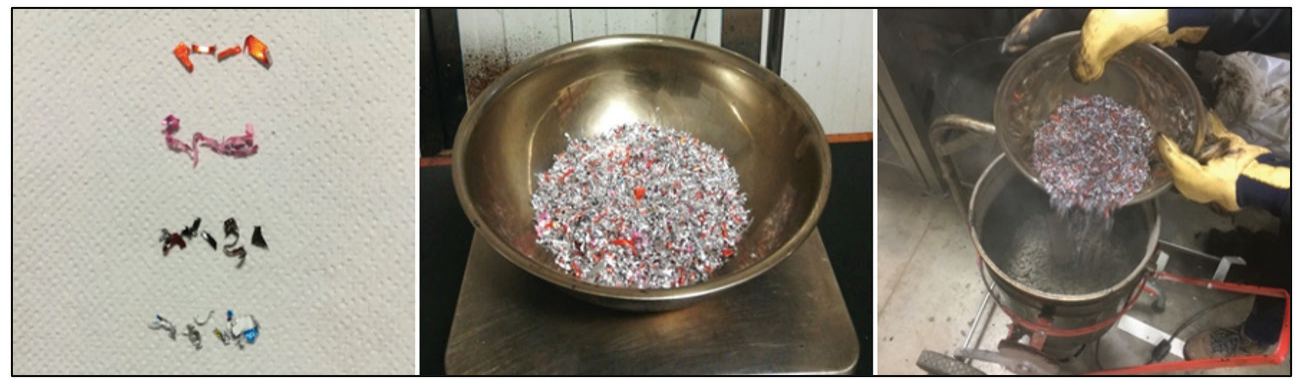

Figura 2. Material de desecho tipo blíster 
Cuadro 1. Propiedades volumétricas de la mezcla control y de las mezclas modificadas con 0,5\%, 1,0\% y 1,5\% de blíster

\begin{tabular}{|c|c|c|c|c|c|}
\hline Propiedad & Control & $0,5 \%$ & $1,0 \%$ & $1,5 \%$ & Requisitos diseño Superpave (CR-2010) \\
\hline PTM $\left.^{1} \%\right)$ & 6,32 & 6,32 & 6,32 & 6,32 & - \\
\hline VTM $^{2}(\%)$ & 4,00 & 4,30 & 4,40 & $5,30^{*}$ & - \\
\hline VMA $^{3}(\%)$ & 14,94 & 14,90 & 14,90 & 15,30 & $>14$ \\
\hline VFA $^{4}(\%)$ & 73,31 & 71,20 & 70,40 & 65,60 & $65-75$ \\
\hline P/A $^{5}$ & 1,19 & 1,00 & 1,01 & 1,05 & $0,6-1,3$ \\
\hline
\end{tabular}

1 PTM (\%): Porcentaje de asfalto por peso total de la mezcla

${ }^{2}$ VTM (\%): Porcentaje total de vacíos de la mezcla

${ }^{3}$ VMA (\%): Porcentaje de vacíos en el agregado mineral

${ }^{4}$ VFA (\%): Porcentaje de vacíos rellenos de asfalto

${ }^{5} \mathrm{P} / \mathrm{A}$ : Relación polvo asfalto

* No cumple con el porcentaje de diseño teórico de vacíos de la metodología Superpave $(4 \pm 1) \%$

A pesar de que la mezcla con $0,5 \%$ presentó un porcentaje de vacíos más cercano al teórico, se decidió utilizar el 1,0 \% como porcentaje óptimo para el proyecto; ya que, además de cumplir con los requisitos de diseño de la metodología Superpave, permitió utilizar una mayor cantidad de modificante, dándole un mayor uso a este desecho. Mientras que, si se utilizaba un porcentaje más alto de modificante como lo es el 1,5 \%, el volumen total de vacíos se alejaba del teórico.

Al tener el diseño establecido, se procedió a elaborar los especímenes de ensayo con el propósito de determinar su desempeño y comparar los resultados con los de la mezcla control. Los ensayos utilizados para realizar la comparación del comportamiento mecánico de las mezclas fueron: fatiga a flexotracción en cuatro puntos, rueda de Hamburgo, resistencia retenida a la tensión diametral y módulo dinámico.

Para realizar los especímenes, se calculó la cantidad de mezcla requerida, definiendo los pesos de cada material (agregado, asfalto y material de desecho). Conociendo esta información, fue necesario, previo a la elaboración de los especímenes, asegurarse que los agregados utilizados en la mezcla no presentaran humedad, por lo que se colocó el agregado de Río Claro al horno durante $16 \mathrm{~h}$ a una temperatura de $110^{\circ} \mathrm{C}$.

La mezcla se realizó en una mezcladora con agitación mecánica, la cual debía de llevarse a temperatura de mezclado con el propósito de evitar que la mezcla se adhiriera al equipo y se diera un desperdicio de material (proceso que se debió realizar para cada utensilio que estuviera en contacto con la mezcla). A la vez, se debió llevar el asfalto a $162{ }^{\circ} \mathrm{C}$ y los agregados a $180{ }^{\circ} \mathrm{C}$, para asegurar que se mantuvieran en los rangos de temperatura de mezclado $\left(160{ }^{\circ} \mathrm{C}\right)$.
La temperatura del agregado es mayor, contemplando la pérdida de temperatura al momento de sacarlos del horno y preparar el lote de la mezcla.

Una vez preparados los materiales se procedió a la elaboración de la mezcla modificada, colocando inicialmente el agregado grueso, seguido del material de desecho tipo blíster. Ambos materiales se mezclaron $y$, posteriormente, se incorporó el agregado fino. Al mezclarse estos agregados, se añadió el asfalto y se mezcló hasta lograr una mezcla homogénea. El tiempo de mezclado para la mezcla modificada no difirió con respecto al de la mezcla convencional.

Seguidamente, se curó la mezcla, método que consiste en colocar la mezcla a temperatura de compactación $\left(152{ }^{\circ} \mathrm{C}\right)$ por $2 \mathrm{~h}$ para que el agregado finalice de absorber el asfalto. Al concluir este periodo, se procedió a compactar los especímenes. Para las muestras del ensayo de fatiga en cuatro puntos se requirió del equipo Asphalt Shear Box Compactor y para el módulo dinámico, rueda de Hamburgo y resistencia retenida a la tensión diametral, el Compactador Giratorio Superpave (los moldes de estos equipos debían estar a temperatura de compactación).

Los especímenes se compactaron hasta alcanzar el porcentaje de vacíos requeridos. Siendo este de $(7 \pm 1) \%$ para los destinados a rueda de Hamburgo y resistencia retenida a la tensión diametral. Y de $8 \%$ para los de módulo dinámico y fatiga a cuatro puntos; con el propósito de asegurar que al ser cortados (como se especifica en las normas) alcanzaran el $(7 \pm 1) \%$. Al finalizar la elaboración de dichos especímenes se procedió a realizar la falla de las muestras. En la Figura 4 se muestran los especímenes realizados para cada ensayo. 


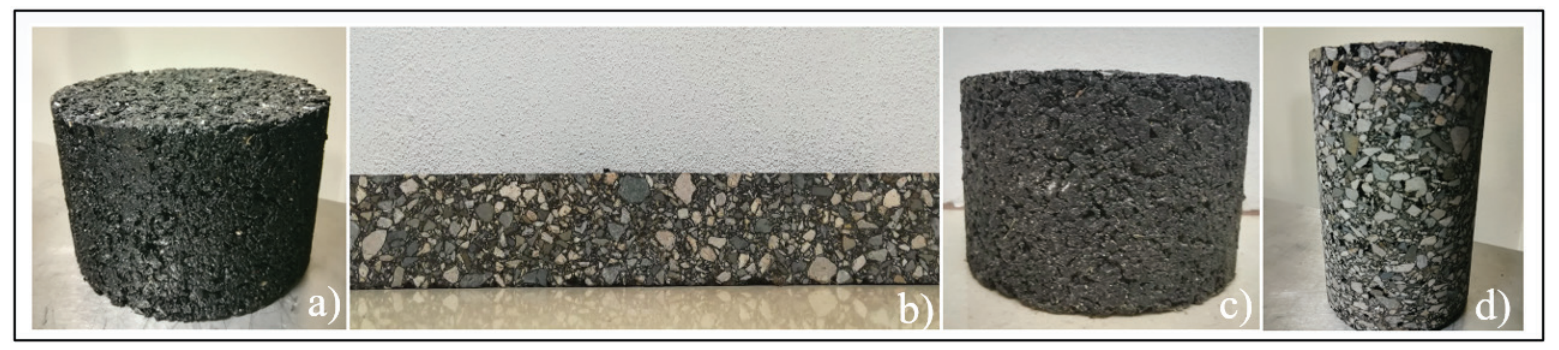

Figura 4. Especímenes elaborados con la mezcla asfáltica modificada para las pruebas de a) rueda de Hamburgo; b) fatiga a cuatro puntos; c) tensión diametral; d) módulo dinámico

\section{RESULTADOS}

\section{Caracterización del modificante}

Para asegurar una correcta incorporación del material modificante, es de suma importancia tener identificado la temperatura de degradación del material, ya que esta debe ser mayor a la temperatura de modificación y de mezclado (Villegas y Aguiar, 2013).

Debido a esto, se caracterizó el material con las pruebas de: espectroscopia infrarroja por transformada de Fourier (FTIR), análisis termogravimétrico (TGA) y calorimetría de barrido diferencial (DSC).

El ensayo de FTIR permite caracterizar materiales por medio de espectros infrarrojos que muestran señales de absorción de la muestra analizada. Al analizar los espectros infrarrojos obtenidos de las muestras de blíster, se asoció con diferentes polímeros: polimetacrilato, cloruro de polivinilo (PVC) y polietileno de baja densidad (LDPE).

Por medio de la prueba del TGA, se logra determinar la estabilidad térmica del material y su temperatura de descomposición. Para el caso del blíster se pudo observar que antes de los $200^{\circ} \mathrm{C}$ la muestra presenta pérdidas de humedad y de volátiles livianos; y entre los $200^{\circ} \mathrm{C}$ y los $800^{\circ} \mathrm{C}$ ocurre la mayor descomposición del material.

Por otro lado, la técnica del DSC permite identificar las transiciones térmicas (fusión, cristalización y transiciones vítreas) del material analizado. Con esta prueba se pudo identificar que el blíster experimenta transiciones vítreas entre $\operatorname{los} 50^{\circ} \mathrm{C}$ y los $100{ }^{\circ} \mathrm{C}$. Y entre los $90{ }^{\circ} \mathrm{C}$ y los $120{ }^{\circ} \mathrm{C}$, presenta un proceso endotérmico asociado a la fusión del material.

Con los resultados obtenidos se pudo determinar que a temperaturas de mezclado $\left(160^{\circ} \mathrm{C}\right)$ no se da la degradación del material, ni un cambio de fase que dificulte su mezclado, de esta forma se evalúa que el blíster sea apto para la modificación de mezcla asfáltica a las condiciones de mezclado y colocación.

\section{Desempeño de la mezcla asfáltica modificada}

\section{Fatiga a cuatro puntos}

La prueba de fatiga a cuatro puntos (AASHTO T 321-17) consiste en someter a flexotracción repetidamente una viga con el propósito de alcanzar la fatiga y determinar los ciclos de falla (AASHTO, 2017). Para la mezcla control y modificada, el ensayo se realizó a deformaciones controladas, 400 micro strain y 600 micro strain y a una temperatura de $20^{\circ} \mathrm{C}$. Esto con el propósito de verificar el cumplimiento de los requisitos de desempeño y aceptación de diseño, del Manual de especificaciones generales para la construcción de carreteras, caminos y puentes de Costa Rica (CR-2010), establecidos en la sección 402, donde se detallan las especificaciones para el diseño de una mezcla asfáltica utilizando el método Superpave.

En el Cuadro 2 se pueden observar los resultados promedio de la rigidez inicial, de la rigidez en el ciclo de falla y de los números de ciclos alcanzados para llegar a la falla, de ambas mezclas bajo las dos deformaciones unitarias analizadas (en el Cuadro se resaltan los valores más altos entre los resultados de la mezcla control y la modificada para cada estado de deformación).

Cuadro 2. Resultados promedio del ensayo de fatiga a deformación controlada

\begin{tabular}{|c|c|c|c|c|}
\hline Deformación & \multicolumn{2}{|c|}{400 micro strain } & \multicolumn{2}{c|}{600 micro strain } \\
\hline Mezcla & Modificada & Control & Modificada & Control \\
\hline Rigidez inicial (MPa) & $\mathbf{7 2 9 6}$ & 6513 & $\mathbf{6 8 8 1}$ & 6326 \\
\hline Rigidez en ciclo de falla (MPa) & 2955 & $\mathbf{3 3 8 0}$ & 1991 & $\mathbf{3 2 6 4}$ \\
\hline No Ciclos para la falla & $\mathbf{5 2 4 0 7 7}$ & 190149 & $\mathbf{5 9 2 9 5}$ & 19461 \\
\hline
\end{tabular}


Los datos reflejan que, para ambas deformaciones controladas, la mezcla modificada presenta una rigidez inicial mayor. Además de una mayor pérdida de rigidez, lo que se asocia a que esta mezcla requiera de una mayor cantidad de ciclos para alcanzar la falla.

Al realizar una comparación de las medias de los ciclos de falla según el tipo de mezcla y la deformación controlada, por medio de un análisis estadístico tipo ANOVA con un nivel de confianza de $95 \%$, se logró concluir que el tipo de mezcla y la deformación afectan los ciclos de falla, y que entre estas variables existe una dependencia. Es decir, el cambio relacionado con la deformación es función del tipo de mezcla.

Por otro lado, se observa que la mezcla modificada es más susceptible a cambios en las deformaciones unitarias generadas por cargas, comportamiento que puede verse reflejado en la Figura 5. Donde también se ilustra que la mezcla modificada posee un comportamiento óptimo a fatiga para los estados de deformación analizados; ya que, para alcanzar un mismo estado de deformación, la mezcla requiere una mayor cantidad de ciclos.

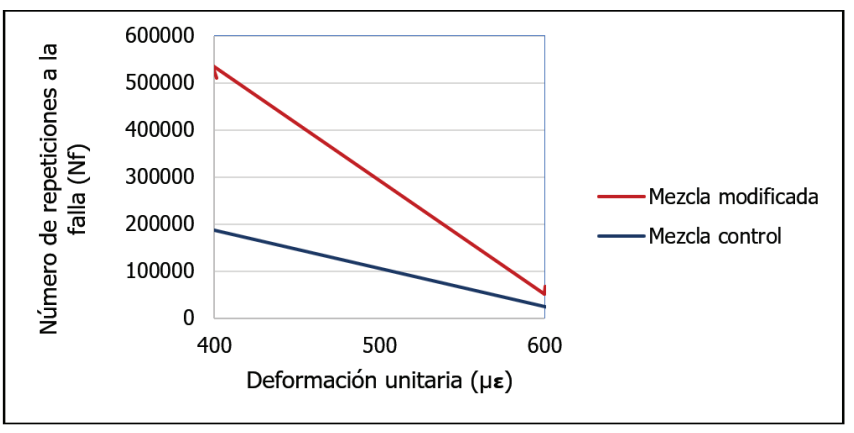

Figura 5. Deformación unitaria contra número de repeticiones a la falla

Se debe recalcar que según las especificaciones del CR-2010, la mezcla modificada cumple los ciclos promedio para la falla por fatiga para los niveles de deformación unitaria controlada en mezclas asfálticas de alto desempeño (mezcla $C$ aplicadas en capas asfálticas intermedias y mezcla D aplicadas en capas asfálticas de rodadura, ambas para un alto volumen de tránsito, es decir, más de 10 millones ESAL's), mientras que la de control no lo hace.

\section{Módulo dinámico}

El ensayo de módulo dinámico (ASTM D3496 y D3497, AASHTO TP62) permite determinar las propiedades elásticas de la mezcla. La prueba consiste en aplicarle al espécimen, a temperaturas y frecuencias definidas, un esfuerzo de compresión axial sinusoidal y la deformación asociada a este esfuerzo es la que permite calcular el módulo dinámico.

Los módulos dinámicos obtenidos de la mezcla analizada pueden ser utilizados para elaborar una curva maestra, la cual permite identificar el desempeño de las mezclas a diferentes frecuencias y a una temperatura de referencia $\left(21,1^{\circ} \mathrm{C}\right.$ para el presente estudio). Las curvas maestras de las mezclas control y modificada se pueden observar en la Figura 6.

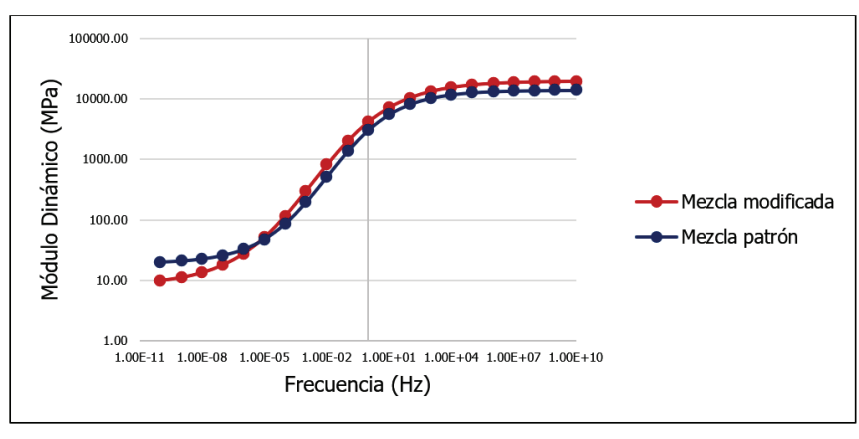

Figura 6. Curvas maestras de la mezcla control y la mezcla modificada

En las curvas maestras se ve que la mezcla modificada presenta mayoritariamente módulos superiores, reflejando una menor deformación bajo un mismo estado de esfuerzo. Por otro lado, se identifica que a frecuencias muy altas la mezcla modificada presenta módulos superiores a la de control, pero a frecuencias muy bajas el comportamiento se invierte. Específicamente, en las colas de estas curvas la diferencia es alta, a una frecuencia de 1,00E-10 la mezcla modificada posee $51 \%$ menos rigidez que la de control y a 1,00E10, $40 \%$ más rigidez. No obstante, los módulos obtenidos en las colas de las curvas no son exactos; ya que, los valores de los extremos de la curva son una extrapolación de los resultados obtenidos en el ensayo. Por lo que estas diferencias no son de tanta relevancia para la comparación entre las mezclas.

Se realizó además una comparación estadística (por medio de un ANOVA con un nivel de confianza de 95 \%) de los módulos dinámicos obtenidos en cada mezcla, considerando tres factores: tipo de mezcla, temperatura y frecuencia. De este análisis se concluyó que los tres factores afectan el valor del módulo dinámico, provocando que las medias de los módulos difieran. A la vez, se dedujo que el módulo dinámico depende de la interacción entre la temperatura y la frecuencia, y de la interacción de la temperatura y el tipo de mezcla. 


\section{Rueda de Hamburgo}

El ensayo de Rueda de Hamburgo (AASHTO T 324-19) permite determinar la susceptibilidad a la deformación permanente y al daño por humedad. Para la prueba, los especímenes se sumergen en agua y se someten a una carga repetitiva. La deformación se define con la profundidad de impresión (rutina) la cuál se manifiesta según los números de pasadas de la rueda. Y la falla asociada al daño por humedad se determina según el punto de inflexión, el cual está relacionado con la cantidad de ciclos para llegar a la falla; al ser este valor más alto, mejor es la mezcla. El ensayo finaliza cuando la rutina llega su máximo $(20 \mathrm{~mm})$ o cuando se alcanzan 20000 ciclos.

En el Cuadro 3 se detallan los resultados de esta prueba. La mezcla modificada presenta una impresión máxima promedio menor a la profundidad de falla de $20 \mathrm{~mm}$, por lo que el ensayo continuó hasta alcanzar los 20000 ciclos. Mientras que la mezcla control, alcanzó un valor promedio de la impresión máxima superior a la profundidad de falla, finalizando en este punto el ensayo, que fue aproximadamente a los 10000 ciclos, siendo esta la mitad de ciclos alcanzados por la mezcla modificada. De igual manera, para la mezcla control la cantidad de ciclos reportados para alcanzar el punto de inflexión fue aproximadamente la mitad de los de la mezcla modificada, reflejando un mejor comportamiento de la mezcla modificada.

\section{Resistencia retenida a la tensión diametral (RRTD)}

Con el ensayo de RRTD (AASHTO T283, ASTM D4867) se logra determinar la susceptibilidad al daño por humedad, evaluando el impacto del agua en la resistencia a la tracción de la mezcla. El ensayo consiste en dividir los especímenes en dos condiciones, seca y parcialmente saturada. A estos especímenes se les aplica una carga y se registra la máxima soportada. Con estos valores, se calcula el porcentaje de resistencia de muestras secas, que alcanzan los especímenes saturados.

En el Cuadro 4 se muestran los resultados de este ensayo para las mezclas analizadas (en el cuadro se resaltan los valores más altos entre los resultados de la mezcla control y la modificada). Para ambas condiciones, la mezcla modificada presenta un promedio de carga máxima superior. Además, se puede notar que el promedio de la resistencia retenida a la tensión diametral es mayor en la mezcla modificada.

Cuadro 3. Impresión máxima y cantidad de ciclos del punto de inflexión

\begin{tabular}{|c|c|c|c|c|c|}
\hline Mezcla & Grupo & $\begin{array}{l}\text { Impresión máxima } \\
\qquad(\mathrm{mm})\end{array}$ & $\begin{array}{l}\text { Número de } \\
\text { pasadas }\end{array}$ & $\begin{array}{l}\text { Profundidad de falla } \\
\qquad(20 \mathrm{~mm})\end{array}$ & Ciclos del punto de inflexión \\
\hline \multirow{3}{*}{ Modificada } & Llanta izquierda & $-20,45$ & 19950 & Falla & \multirow{3}{*}{12785} \\
\hline & Llanta derecha & $-12,66$ & 19950 & Pasa & \\
\hline & Promedio & $-16,55$ & - & Pasa & \\
\hline \multirow{3}{*}{ Control } & Llanta izquierda & $-20,03$ & 10005 & Falla & \multirow{3}{*}{5929} \\
\hline & Llanta derecha & $-20,10$ & 10579 & Falla & \\
\hline & Promedio & $-20,06$ & - & Falla & \\
\hline
\end{tabular}

Cuadro 4. Resultados de resistencia retenida a la tensión diametral

\begin{tabular}{|c|c|c|}
\hline \multicolumn{2}{|c|}{ Condición Seca } & Control \\
\hline Mezcla & Modificada & 15048 \\
\hline Promedio de carga máx (N) & $\mathbf{2 1 1 5 6}$ & Control \\
\hline Mezcla & Condición Saturada & 75,7 \\
\hline Promedio del \% Saturación & Modificada & 12573 \\
\hline Promedio de carga máx (N) & $\mathbf{7 6 , 7}$ & 84,0 \\
\hline Promedio RRTD (\%) & $\mathbf{1 8 7 2 9}$ & $\mathbf{8 8 , 6}$ \\
\hline
\end{tabular}


Sin embargo, se realizó un ANOVA a un $95 \%$ de confianza para determinar si las medias de los porcentajes de resistencia retenida de las mezclas diferían. De este análisis, se concluyó que no existe estadísticamente una diferencia entre la resistencia de la mezcla control y la de la mezcla modificada. Sin embargo, es importante recalcar que si se analiza este valor con las especificaciones del CR-2010, la mezcla modificada cumple con las especificaciones para mezclas de alto desempeño y la de control, no.

\section{Evaluación de sensibilidad de la mezcla modificada}

La evaluación de la sensibilidad de la mezcla se basó en los deterioros estructurales y en la degradación del blíster. Como se mencionó anteriormente, el blíster está compuesto por un conjunto de polímeros (principalmente PVC), por lo que se identificaron los tipos de degradaciones que podrían afectar este material; siendo estos la degradación térmica y la degradación fotoquímica.

La degradación térmica se da a altas temperaturas, y provoca una separación de las cadenas del polímero. Este proceso ocurre a dos temperaturas, la inicial $\left(\mathrm{T}_{\mathrm{d}}\right)$ y la media $\left(\mathrm{T}_{\mathrm{m}}\right)$. En la temperatura inicial se pierde el $10 \%$ de las propiedades físicas y en la temperatura media $\left(\mathrm{T}_{\mathrm{m}}\right)$ se pierde el $50 \%$ de su peso luego de ser calentado por $30 \mathrm{~min}$. Para el polímero tipo PVC la $\mathrm{T}_{\mathrm{d}}$ ocurre a los $170{ }^{\circ} \mathrm{C}$ y la $\mathrm{T}_{\mathrm{m}}$ a los $279^{\circ} \mathrm{C}$ (Carranza, et al., 2010). Es decir, el blíster al estar compuesto principalmente por este polímero, no se vería afectado por este tipo de degradación a la hora de producir y colocar la mezcla.

No obstante, el blíster expuesto a rayos ultravioleta es susceptible a la degradación fotoquímica, degradación en la que se da la separación molecular por la incidencia de radiación electromagnética. Generalmente, la radiación ultravioleta se da entre los $300 \mathrm{~nm}$ y los $400 \mathrm{~nm}$, y los polímeros sintéticos puros (como el PVC) no absorben longitudes mayores a los $300 \mathrm{~nm}$. Sin embargo, la radiación solar puede deteriorar el material por presencia de impurezas o defectos en su estructura (Carranza, et al., 2010).

Con los resultados obtenidos de los ensayos de desempeño, se pudo observar que la mezcla modificada es menos susceptible a la deformación permanente, que el daño por humedad se alcanza a mayores ciclos en la mezcla modificada y que esta resiste mayores cargas, por lo que esta mezcla tiene características aptas para ser colocada en capas superficiales. Se debe resaltar que, según un estudio elaborado por el LanammeUCR, la fuente de agregado utilizada en el diseño presenta alta susceptibilidad al daño por humedad, comparado con otras fuentes utilizadas en el país (Lanamme, 2017).

Por otro lado, se pudo observar que la deformación alcanzada por la mezcla modificada es inferior a la de la mezcla control ante un mismo estado de esfuerzo y que la mezcla modificada, requiere mayor cantidad de ciclos de carga para alcanzar la falla por fatiga. Con estos resultados, se recomienda utilizar la mezcla modificada en capas inferiores. También se determinó que la modificada cumplió con las especificaciones del CR-2010 (MOPT, 2017) de mezclas de alto desempeño, por lo que podría utilizarse para carreteras con alto tránsito.

Finalmente, es importante recalcar que al utilizar la mezcla asfáltica modificada con $1 \%$ de blíster (cuya densidad es de $2,4 \mathrm{t} / \mathrm{m}^{3}$ ) en proyectos de infraestructura vial, se estaría aportando a reducir la contaminación y a ayudar al medio ambiente, ya que se utilizaría gran proporción del desecho en la mezcla, por lo que se le estaría dando un uso al blíster que actualmente posee pocas alternativas para ser reutilizado o reciclado. Por ejemplo, en un kilómetro de carretera, con dos carriles de 3,6 $\mathrm{m}$ de ancho cada uno y un espesor de $5 \mathrm{~cm}$, se necesitarían 863 t de mezcla; de las cuales 8 t serían de blíster.

\section{CONCLUSIONES}

La mezcla asfáltica modificada con material de desecho tipo blíster bajo las condiciones y la dosificación utilizadas presentó mejores resultados que la mezcla convencional. Además, la granulometría utilizada con el desecho presentó una buena trabajabilidad y se obtuvo una mezcla homogénea.

A la vez, la mezcla modificada reflejó una mejora en el desempeño comparado con la mezcla control, la modificada presentó mayor resistencia a la fatiga, a la humedad y a la deformación permanente. Específicamente en cuanto a la fatiga, la mezcla modificada requirió de más ciclos para alcanzar la falla, y estos ciclos dependen del estado de deformación, del tipo de mezcla y de la interacción entre estos factores. Además, la mezcla modificada presentó mayor sensibilidad ante los cambios en la deformación (para las deformaciones controladas en estudio).

Por otro lado, se determinó que la mezcla modificada registra módulos dinámicos superiores a frecuencias altas, traducido en menos deformación bajo un mismo estado de esfuerzo. Y se identificó que los módulos varían según la temperatura, la frecuencia, el tipo de mezcla y según la interacción entre la temperatura y la frecuencia, y entre la temperatura y el tipo de mezcla. 
La mejora en la susceptibilidad al daño por humedad se vio reflejada en una mayor cantidad de ciclos para llegar a la falla, siendo la de la mezcla modificada el doble que la de control.

Por otro lado, a pesar de que estadísticamente la resistencia retenida a la tensión diametral no difiere entre las mezclas, la mezcla modificada puede ser utilizada como mezcla de alto desempeño, debido a que cumple con las especificaciones del CR-2010 en la resistencia retenida a la tensión diametral (al igual que con la fatiga) y la mezcla control no las cumple.

Así mismo, se logró identificar que, aunque la degradación térmica no afecta el blíster, este material podría verse afectado por los rayos ultravioleta, en caso de quedar expuesto (no recubierto) en la mezcla. No obstante, analizar la ocurrencia de la foto-degradación del material se sale del alcance de esta investigación.
Además, se notó que, a pesar de que la mezcla modificada resultó ser menos susceptible al daño por humedad y a la deformación permanente, está elaborada con un agregado que es altamente susceptible al daño por humedad, por lo que se debe considerar este factor para colocar esta mezcla en capas superficiales. Por otro lado, si se desea colocar en capas inferiores se debe tomar en cuenta el buen resultado que presentó a fatiga.

Este estudio permitió identificar que el blíster es apto para ser utilizado en proyectos de infraestructura vial, generando una alternativa de reutilización y promoviendo los pavimentos verdes en el país.

En general, el proyecto permite profundizar en el tema de la incorporación de este desecho en mezclas asfálticas. Sin embargo, es importante recalcar que los resultados obtenidos son exclusivamente para el diseño utilizado. A la vez, como la modificación se realizó a escala de laboratorio, se recomienda discreción, ya que los resultados podrían diferir al implementarlos en escala real.

\section{REFERENCIAS}

AASHTO. (2017). AASHTO T 321-17: Standard Method of Test for Determining the Fatigue Life of Compacted Asphalt Mixtures Subjected to Repeated Flexural Bending. United States.

Aguiar, J., Villegas, R., Loría, L., y Salazar, J. (2013). Use of waste products as bitumen modifiers in Costa Rica. EATA Conference. Recuperado de: https://www.lanamme.ucr.ac.cr/repositorio/handle/50625112500/535

Asamblea Legislativa, República de Costa Rica. (2020). Ley $N^{\circ}$ 9828. Ley (Uso de materiales reutilizables en pavimentos y obras de infraestructura vial). Versión de la norma 1 de 1, 21 mayo 2020. Gaceta No. 148 publicada el 21 junio 2020. Recuperado de: http://www.pgrweb.go.cr/scij/Busqueda/Normativa/Normas/nrm_texto_completo.aspx?param1=NRTC\&nValor1=1 \&nValor2=91662\&nValor3=121125\&strTipM=TC

Ávila, J. (2020). Diseño de un proceso de recuperación de aluminio por fusión de los empaques tipo blíster. (Tesis de grado). Escuela Politécnica Nacional, Quito, Ecuador.

Cajina, N. (2020). Diseño y evaluación de desempeño de la mezcla asfáltica modificada con material de desecho. (Tesis de grado). Universidad de Costa Rica, San José, Costa Rica.

Camacho, C. (17 de febrero 2013). PVC, un plástico incómodo de reciclar. El Financiero CR. Recuperado de https://www. elfinancierocr.com/negocios/pvc-un-plastico-incomodo-de- reciclar/EYFG46ASWFDQ3KPHCYHECEYBZU/story/

Caro, S. y Álvarez, A. (2011). Evaluación de la susceptibilidad al daño por humedad de mezclas asfálticas empleando propiedades termodinámicas. Revista Facultad de Ingeniería Universidad de Antioquia, 58, 95-104.

Carranza, R., Duffo, G. y Farina, S. (2010). Nada es para siempre. Química de la degradación de los materiales. Recuperado de http://www.bnm.me.gov.ar/giga1/documentos/EL001847.pdf

Grajales, I. (5 de Junio del 2018). Costa Rica tira al mar 15 camiones de plástico por día. Hoy en el TEC. Recuperado de https:// www.tec.ac.cr/hoyeneltec/2018/06/05/costa-rica-tira-mar-15-camiones-plastico-dia\#: :text=De\%20acuerdo\%20con\%20 el\%20Programa,toneladas\%20de\%20pl\%C3\%A1stico\%20por\%20d\%C3\%ADa.\&text=A\%20pesar\%20de\%20ello\%2C\%20 el,camiones\%20de\%20pl\%C3\%A1stico\%20por\%20d\%C3\%ADa 
Lanamme (2016). Informe de Ensayo para el proyecto: Daño por humedad, fase N3 (I-1264-16).

Lanamme. (2017). Daño por humedad en mezclas asfálticas. (LM-PI-UMP-044-R4).

Mendoza, D.; Ruiz, D. y Cantillo, S. (2019). Automatización de equipo de pista para la evaluación de deformación permanente en mezclas asfálticas. (Tesis de grado). Universidad Piloto de Colombia, Bogotá, Colombia.

MOPT. (2017). Repositorio Sección 402) Diseño de mezcla asfáltica por el método superpave. Recuperado de http://repositorio. mopt.go.cr:8080/xmlui/123456789/3601

Muñoz, M. (2020). Evaluación de procedimientos de ensayo y análisis de la influencia del envejecimiento en la resistencia al agrietamiento por fatiga en mezclas asfálticas en laboratorio. (Tesis de grado). Universidad de Costa Rica, San José, Costa Rica.

Rodríguez, E. (2016). Uso de polvo de caucho de llantas en pavimentos asfálticos. Boletín técnico PITRA-LanammeUCR. 7(4), 1-7. Recuperado de https://www.lanamme.ucr.ac.cr/repositorio/handle/50625112500/316

Rondón, H., Moreno, L., Rodríguez, D. y Lee, J. (2009). Experiencias sobre el estudio de materiales alternativos para modificar asfaltos. Ingeniería, 14(2), 19-33.

Villegas, R. y Aguiar, J. (2013). Metodología integral de incorporación eficaz de modificantes en matrices asfálticas. Recuperado de https://www.lanamme.ucr.ac.cr/repositorio/handle/50625112500/542 